\title{
EDITORIAL
}

\section{Toward a universal influenza vaccine: a retrospective}

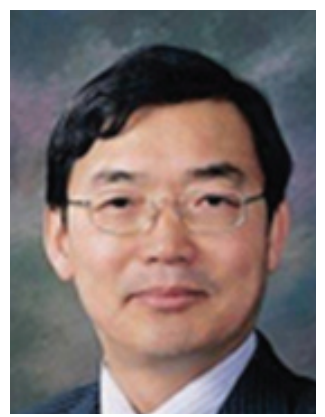

\author{
“Developing a flu vaccine that offers universal \\ protection against all influenza strains is ... \\ tantamount to the quest for the Holy Grail.”
}

Yo Han Jang ${ }^{1} \&$ Baik Lin Seong ${ }^{* 1,2}$

First draft submitted: 3 February 2016; Accepted for publication: 11 February 2016; Published online: 15 April 2016

The influenza virus, due to its segmented nature and intrinsically high mutation rate, is prone to frequent antigenic drifts and antigenic shifts, leading to annual episodes, as well as occasional pandemic outbreaks. Influenza A viruses are broadly categorized into subtypes according to their surface glycoproteins hemagglutinin (HA) and neuraminidase (NA), while the 18 different HA subtypes are further classified into two distinct phylogenetic groups [1] Vaccination has been considered the most effective way to control the influenza virus, but the seasonal influenza vaccines currently available only provide strain-specific protection. Every year, international health authorities including the WHO and the CDC struggle to recommend the HA and NA viral surface antigens to be included in the vaccine. Unfortunately, the empirical nature of the recommendations, which basically amount to an educated guess, inevitably results in occasional vaccine mismatches [2]. Developing a flu vaccine that offers universal protection against all influenza strains is thus tantamount to the quest for the Holy Grail. Accordingly, in recent years, considerable efforts have been made to develop a universal influenza vaccine that provides broad-spectrum protection against diverse influenza strains by eliciting immune responses to conserved domains.

The extracellular domain of the M2 ion channel (M2e) shows a high level of conservancy across all influenza A viruses and hence has been a primary target for the development of a universal influenza vaccine [3]. Subsequently, different types of M2e-based influenza vaccines have been devised and tested for the breadth of protection in animals and humans, in the forms of recombinant protein, DNA and virus-like particles [4]. Although the M2ebased approaches offer a certain level of broad protection, a general observation is that the M2e-based vaccine provides only a weak protection since it is unable to directly

\section{KEYWORDS}

- hemagglutinin stalk • influenza

- universal vaccine

“...in recent years, considerable efforts have been made to develop a universal influenza vaccine that provides broad-spectrum protection against diverse influenza strains by eliciting immune responses to conserved domains." 
“...given the huge variability of the influenza virus genome, a promising vaccine design should consider including as many 'protective and conserved' antigens as possible, while minimizing 'nonprotective' or even 'proinfective' non-neutralizing antibody responses." inhibit the viral entry into the cell. It has also been reported that the protection conferred by the M2e-based vaccine are based largely on indirect antibody-dependent effector mechanisms, such as antibody-dependent cell-mediated cytotoxicity, complement-dependent cytotoxicity and antibody-dependent cell-mediated phagocytosis [3,4]. Still, it is expected that the M2e may contribute to enhancing the overall protection potency if included as part of a universal influenza vaccine construct consisting of multiple conserved epitopes or domains. Another attractive target in the development of a universal influenza vaccine is the HA stalk (stem) domain that mediates the fusion of viral and endosomal membranes and the subsequent uncoating and release of viral genome into the cytoplasm. Most influenza vaccines contain both HA and NA components and antibodies against $\mathrm{HA}$ are particularly important in effectively neutralizing the virus by blocking the host cell attachment and subsequent viral entry. The HA antigen is composed of two major domains, the HAl globular domain, which is highly divergent among various subtypes and drifting strains, and HA2 stalk domain, which is highly conserved. Thus, the stalk-reactive antibodies demonstrate a broader range of protection than the head-reactive antibodies, which provides only strain-specific protection. In contrast to the head-reactive antibodies, which avert viral entry into the cell only by preventing the interaction between the viral HA and the cellular receptor sialic acid residues, the stalk-reactive antibodies exhibit anti-influenza activities through various mechanisms. The stalk-reactive antibodies inhibit $\mathrm{pH}$-dependent conformational changes of the N-terminal fusion peptide of the HA2 subunit and block the membrane fusion event in the cellular endosomes [5,6]. In addition, the stalk-reactive antibodies bind to the uncleaved HA0 precursor and inhibit its protease-mediated processing into HA1 and HA2 subunits, which is an essential step for acquiring viral infectivity [7]. It was also shown in vivo that similar to the M2especific antibodies, the stalk-reactive antibodies cause the killing of the infected cells through the antibody-dependent cell-mediated cytotoxicity and complement-dependent cytotoxicity mechanisms $[8,9]$.

Significant advances were made toward the development of a universal influenza vaccine by rational vaccine designs redirecting the usual immune responses to the variable HA1 globular domain into the conserved HA2 stalk domain.
A prime-boost regimen using the 'headless and stalk only HA' or 'chimeric HA' vaccine constructs has shown that the stalk-reactive antibodies enable the boosting of the antibodies that neutralize both the homologous and heterosubtypic influenza viruses in vitro [10]. These approaches have been extensively tested in animals and found to confer a broad protection against the heterosubtypic influenza viruses within the same HA group viruses. Subsequently, various vaccination technologies were devised and evaluated for their ability to boost the stalk-reactive antibodies, in the forms of recombinant proteins, DNA vaccines, virus-like particles, viral vectors and live attenuated vaccines, either alone or in combinations [10]. In parallel with the vaccination approach for broad protection, particular attention has also been given to the therapeutic potential of the stalk-reactive monoclonal antibodies (mAbs). While most stalkreactive $m A b s$ show cross-reactivity within only one of the HA groups [5,11-12], some rare mAbs exhibited much broader binding specificity covering both HA groups within the influenza $A$ virus and even across both influenza $\mathrm{A}$ and $\mathrm{B}$ viruses [13,14]. Although the binding specificity of the mAbs does not necessarily guarantee the neutralizing activity against the viruses, these results together provide auspicious grounds to expect a truly universal influenza vaccine developed through a rational vaccine design that covers all influenza $A$ viruses or even influenza $A$ and $B$ viruses.

Although the HA stalk-based universal vaccine strategies provide a much broader protection against heterologous and heterosubtypic strains than the classical inactivated vaccines, some important issues remain to be addressed, especially on their effectiveness and safety. Of note, it has been reported that the stalk-reactive antibodies increase the viral infectivity upon challenge infection by heterosubtypic influenza viruses, rather than protecting against them [15]. Although this phenomenon has not yet been observed in the current stalk-based vaccine approaches, close attention should be given to these potential safety issues until the detailed underlying molecular mechanisms are clearly defined. Besides the influenza virus, similar antibody-mediated severe side effects, such as antibody-dependent enhancement and vaccine-associated enhancement of respiratory diseases phenomena, have been reported in numerous other viruses, such as the yellow fever virus, dengue virus, HIV, respiratory syncytial virus, hantavirus, Ebola virus, West Nile virus, 
Japanese encephalitis virus and foot-and-mouth disease virus. Thus, the antibody-mediated side effects represent a serious bottleneck for the development of safe vaccines against a very long list of diverse viruses [16]. A common explanation for such side effects is that if the cross-reactive antibodies generated by prior vaccination or infection bind but poorly neutralize the virus, the binding of non-neutralizing antibodies actually facilitates the viral entry into the cells through the well-known process, Fc- $\gamma$ receptor-mediated endocytosis, and increases the viral infectivity [16]. The antibodymediated infection would be much enhanced especially with the prime-boost vaccination that is meant to augment the stalk-specific antibodies. Further detailed studies will be needed to examine the exact extent of 'protective' activity of the stalkspecific antibodies, not just their binding activity against antigenically distinct influenza viruses. The current approaches require multiple doses of vaccination in order to induce protective antibody response, reflecting a relatively low immunogenicity of the stalk region. Indeed, not only the chimeric HA constructs, in which the globular head domain masks the stalk domain, but also the stem only HA constructs had to be administered at least three times to reach desired levels of protection in animals $[17,18]$. Whether the requirement of multiple vaccinations is a viable option for controlling influenza pandemics remains to be discussed. First, the requirement of three or more doses of vaccinations spread across time to induce sufficiently protective immune responses may cancel out the benefits of a timely supply of the vaccines. Second, the current stalk-based universal vaccines provide only partial protection against the heterosubtypic influenza viruses and the limited protective efficacy further decreases as the antigenic distance between the vaccine and infecting virus increases. More specifically, the vaccines based on the stalk of HA group 1 rarely provides protection against the viruses belonging to HA group 2, thus providing only limited coverage of influenza viruses comprising 18 antigenically distant strains [17]. Thus, the degree of heterogeneity of the HA stalk domains between the HA group 1 and 2 influenza viruses belies the intrinsic limitation of the stalk-based universal vaccine approach.

A subdominant immunogenicity of the HA stalk, and a limited protection efficacy and coverage of the stalk-based vaccine strategies together raise a careful question as to whether the stalkspecific antibody immune response alone can provide enough coverage against all influenza $\mathrm{A}$ viruses, let alone allay the potential safety issues associated with the stalk-specific non-neutralizing antibodies. Considering that the influenza virus has continually evolved in multiple ways to counteract the host immune responses and antiviral drugs, the possibility remains that the virus manages to utilize the stalk-specific antibodies as a means to facilitate its infection, counterfeiting the vaccination strategy that was meant to elicit broadspectrum protection. Thus, further considerations should be given to an alternative design of a 'truly' universal influenza vaccine or vaccination strategy with desired levels of protective efficacy, safety and practicality. This ultimate design, from a technical point of view, preferably includes strategies to elicit all phases of immune responses contributing to cross-protection. It has been well known that a number of protective and conserved regions or epitopes that can induce humoral and cellmediated immune responses are embedded in the internal proteins, as well as the surface proteins [19]. In the M2e or HA stalk-based vaccine approaches, cross-protection based on just a small number of conserved epitopes in a single domain is likely to be diluted down to limited protective efficacy and narrow coverage, while also compromising the efficacy by unwanted side effects, such as the antibody-dependent enhancement and vaccineassociated enhancement of respiratory diseases. From a practical point of view, single vaccination is preferable to multiple vaccinations. Whether a live attenuated vaccine, which was shown to provide a broad-spectrum protection to both group 1 and 2 viruses by a single vaccination [20], would serve as a suitable alternative of a universal vaccine, remains to be further investigated in higher animal models and in clinical settings. In summary, given the huge variability of the influenza virus genome, a promising vaccine design should consider including as many 'protective and conserved' antigens as possible, while minimizing 'nonprotective' or even 'proinfective' non-neutralizing antibody responses.

Financial \& competing interests disclosure

This work was supported by the Vaccine Translational Research Center funded by the Ministry of Health and Welfare (HI13C0826), the Korean government. The authors have no other relevant affliations or financial involvement with any organization or entity with a financial interest in or financial conflict with the subject matter or materials discussed in the manuscript apart from those disclosed.

No writing assistance was utilized in the production of this manuscript. 


\section{References}

1 Tong S, Zhu X, Li Y et al. New World bats harbor diverse influenza A viruses. PLoS Pathog. 9(10), e1003657 (2013).

2 Carrat F, Flahault A. Influenza vaccine: the challenge of antigenic drift. Vaccine 25(39-40), 6852-6862 (2007).

3 Deng L, Cho KJ, Fiers W, Saelens X. M2e-based universal influenza A vaccines. Vaccine 3(1), 105-136 (2015).

4 Fiers W, De Filette M, El Bakkouri K et al. M2e-based universal influenza A vaccine. Vaccine 27(45), 6280-6283 (2009).

5 Sui J, Hwang WC, Perez $S$ et al. Structural and functional bases for broad-spectrum neutralization of avian and human influenza A viruses. Nat. Struct. Mol. Biol. 16(3), 265-273 (2009)

6 Ekiert DC, Bhabha G, Elsliger MA et al. Antibody recognition of a highly conserved influenza virus epitope. Science 324(5924), 246-251 (2009).

7 Brandenburg B, Koudstaal W, Goudsmit J et al. Mechanisms of hemagglutinin targeted influenza virus neutralization. PLoS ONE 8(12), e80034 (2013).

8 Dilillo DJ, Tan GS, Palese P, Ravetch JV. Broadly neutralizing hemagglutinin stalk-specific antibodies require Fc $\gamma \mathrm{R}$ interactions for protection against influenza virus in vivo. Nat. Med. 20(2), 143-151 (2014).

9 Jegaskanda S, Weinfurter JT, Friedrich TC, Kent SJ. Antibody-dependent cellular cytotoxicity is associated with control of pandemic H1N1 influenza virus infection of macaques. J. Virol. 87(10), 5512-5522 (2013).

10 Krammer F, Palese P. Advances in the development of influenza virus vaccines. Nat. Rev. Drug Discov. 14(3), 167-182 (2015).

11 Friesen RH, Lee PS, Stoop EJ et al. A common solution to group 2 influenza virus neutralization. Proc. Natl Acad. Sci. USA 111(1), 445-450 (2014).

12 Ekiert DC, Friesen RH, Bhabha G et al. A highly conserved neutralizing epitope on group 2 influenza A viruses. Science 333(6044), 843-850 (2011).

13 Dreyfus C, Laursen NS, Kwaks T et al. Highly conserved protective epitopes on influenza B viruses. Science 337(6100), 1343-1348 (2012).

14 Corti D, Voss J, Gamblin SJ et al. A neutralizing antibody selected from plasma cells that binds to group 1 and group 2 influenza A hemagglutinins. Science 333(6044), 850-856 (2011).
15 Khurana S, Loving CL, Manischewitz J et al. Vaccine-induced anti-HA2 antibodies promote virus fusion and enhance influenza virus respiratory disease. Sci. Transl. Med. 5(200), 200ra114 (2013)

16 Tirado SM, Yoon KJ. Antibody-dependent enhancement of virus infection and disease. Viral Immunol. 16(1), 69-86 (2003).

17 Krammer F, Pica N, Hai R, Margine I, Palese P. Chimeric hemagglutinin influenza virus vaccine constructs elicit broadly protective stalk-specific antibodies. J. Virol. 87(12), 6542-6550 (2013).

18 Impagliazzo A, Milder F, Kuipers $\mathrm{H}$ et al. A stable trimeric influenza hemagglutinin stem as a broadly protective immunogen. Science 349(6254), 1301-1306 (2015).

19 Stanekova Z, Vareckova E. Conserved epitopes of influenza A virus inducing protective immunity and their prospects for universal vaccine development. Virol. J. 7, 351 (2010).

20 Jang YH, Byun YH, Lee YJ, Lee YH, Lee KH, Seong BL. Cold-adapted pandemic 2009 $\mathrm{H} 1 \mathrm{~N} 1$ influenza virus live vaccine elicits cross-reactive immune responses against seasonal and $\mathrm{H} 5$ influenza A viruses. J. Virol. 86(10), 5953-5958 (2012). 